\title{
Regularization of instabilities in gravity theories
}

\author{
Fethi M. Ramazanoğlu \\ Department of Physics, Koç University, Rumelifeneri Yolu, 34450 Sariyer, Istanbul, Turkey
}

(Received 2 October 2017; published 8 January 2018)

\begin{abstract}
We investigate instabilities and their regularization in theories of gravitation. Instabilities can be beneficial since their growth often leads to prominent observable signatures, which makes them especially relevant to relatively low signal-to-noise ratio measurements such as gravitational wave detections. An indefinitely growing instability usually renders a theory unphysical; hence, a desirable instability should also come with underlying physical machinery that stops the growth at finite values, i.e., regularization mechanisms. The prototypical gravity theory that presents such an instability is the spontaneous scalarization phenomena of scalar-tensor theories, which feature a tachyonic instability. We identify the regularization mechanisms in this theory and show that they can be utilized to regularize other instabilities as well. Namely, we present theories in which spontaneous growth is triggered by a ghost rather than a tachyon and numerically calculate stationary solutions of scalarized neutron stars in these theories. We speculate on the possibility of regularizing known divergent instabilities in certain gravity theories using our findings and discuss alternative theories of gravitation in which regularized instabilities may be present. Even though we study many specific examples, our main point is the recognition of regularized instabilities as a common theme and unifying mechanism in a vast array of gravity theories.
\end{abstract}

DOI: 10.1103/PhysRevD.97.024008

\section{INTRODUCTION}

Gravitational physics has been on the forefront of science thanks to the increasing number of gravitational wave detections in recent years [1]. Aside from being a novel observational channel for astrophysics, gravitational waves also enable us to test Einstein's general relativity (GR) for strong and dynamical fields, which has not been possible before now. This led to a resurgence of interest in alternative theories of gravitation [2,3]. Instabilities, degrees of freedom that grow to large values, possibly to infinity, from small perturbations play an important role in alternative theories of gravitation. One prominent example is a tachyonic instability of the scalar field in certain scalartensor theories which causes the zero scalar field solution to be unstable in the presence of neutron stars (NSs). This instability is eventually regularized, i.e., it stops growing at finite field values, and a stable large scalar cloud forms around the star. This phenomenon is named spontaneous scalarization and is particularly interesting to gravitational wave observations. This is due to the large scalar fields causing order-of-unity deviations from GR that are relatively easy to detect with the limited precision of the detectors $[3,4]$.

Despite their eventual regularization in spontaneous scalarization, instabilities can be a nuisance if they are not regulated. Generic interacting spin-2 field theories are well known to possess an instability due to the existence of a ghost field which renders them unphysical. Avoiding the ghost has only recently been possible for carefully constructed theories [5-7], and the ghost can reappear if these constructions are modified, for example, when a nonminimal matter coupling is introduced [8].

Our main aim in this work is to identify some of the cases in which instabilities can be regularized in theories of gravitation. First, this enables us to better understand theories with known regularized instabilities and discover previously unknown cases in the vast landscape of alternative theories of gravitation. The discovery of spontaneous scalarization occurred more than three decades after initial scalar-tensor theories appeared [9], and a better understanding of the underlying physics can make further discoveries faster and more systematic. Second, the understanding of regularization might provide tools to regularize unwanted instabilities that plague many theories of gravitation, such as the interacting spin-2 case.

We start with an analysis of spontaneous scalarization and argue that the type of instability, a tachyon in this case, is irrelevant for the regularization mechanism. Nonlinear terms that become dominant as the scalar field grows are the dominant reason for regularization, while the fact that the tachyon can only live in a finite volume inside the star might also play a part. For an explicit demonstration of this idea, we construct a theory in which a different instability, a ghost rather than a tachyon, leads to spontaneous scalarization. We compute NS solutions in this ghost-based spontaneous scalarization theory, showing that a finite stationary scalarized star solution exists. We also extend these results to spontaneous vectorization [10]. In 
summary, these strongly support the idea that regularization mechanisms of spontaneous scalarization are effective for other instabilities as well. However, proof of the stability of these solutions requires time evolution, including the case of scalar ghost instabilities.

In the final parts of the paper, we qualitatively discuss the possibility of using the aforementioned regularization mechanisms to build a well-behaved theory of spontaneously growing spin-2 fields, which is the natural case after spontaneous scalarization and vectorization, which are already known. This is a theory of interacting spin-2 fields with nonminimal matter couplings as well, which we stated to be ill behaved for generic cases due to the reappearance of the ghost. However, since the regularization mechanisms of spontaneous growth seem to act irrespective of the nature of the instability, we speculate that the reappeared ghost may be rendered harmless by these same mechanisms, leading to a well-behaved theory.

Finally, we shortly discuss further forms of instabilities that might be regularized by similar mechanisms, especially in more general scalar-tensor theories as in Horndeski and beyond-Horndeski theories [11,12]. All these cases show how diverse theories of gravitation are connected in an unexpected way through the role instabilities play in them.

\section{OVERVIEW OF SPONTANEOUS SCALARIZATION}

We start with a short explanation of spontaneous scalarization following [13], with an emphasis on the role of the instability. The action for spontaneous scalarization is [13-15]

$$
\begin{aligned}
& \frac{1}{16 \pi} \int d V R-\frac{1}{16 \pi} \int d V\left[2 g^{\mu \nu} \partial_{\mu} \phi \partial_{\nu} \phi+2 m_{\phi}^{2} \phi^{2}\right] \\
& \quad+S_{m}\left[\psi_{m}, A^{2}(\phi) g_{\mu \nu}\right]
\end{aligned}
$$

where $g_{\mu \nu}$ is the metric, $\phi$ is the scalar field, $m_{\phi}$ is the coupling parameter for the mass potential, and $d V=d^{4} x \sqrt{-g} . S_{m}$ is the matter action, and $\psi_{m}$ denotes the matter degrees of freedom. Without the unusual nonminimal matter coupling, this action simply represents a massive scalar in general relativity, not any alternative theory of gravitation. However, $\psi_{m}$ does not couple directly to the metric $g_{\mu \nu}$ but to a conformally scaled version $\tilde{g}_{\mu \nu}=A^{2}(\phi) g_{\mu \nu}$. This nonminimal coupling breaks the equivalence principle and differentiates this theory from GR. $g_{\mu \nu}$ and $\tilde{g}_{\mu \nu}$ define the so-called Jordan and Einstein frames, respectively. In the following, Jordan frame variables are denoted using tildes to distinguish them from those in the Einstein frame, which are bare.

Let us consider $A(\phi)=e^{\beta \phi^{2} / 2}$ where $\beta$ is a constant parameter of the theory. $\phi=0$ is a trivial solution of the theory and is equivalent to GR. The main point of spontaneous scalarization is that this trivial solution is not necessarily stable, and there is a stable configuration with $\phi \neq 0$ under certain circumstances. That is, any small deviation from $\phi=0$ grows and leads to a nontrivial solution. The reason for the initial growth can be readily seen in the equation of motion (EOM)

$$
\begin{aligned}
\square_{g} \phi & =\left(-8 \pi A^{4} \frac{d(\ln A(\phi))}{d\left(\phi^{2}\right)} \tilde{T}+m_{\phi}^{2}\right) \phi \\
& \approx\left(-4 \pi \beta \tilde{T}+m_{\phi}^{2}\right) \phi,
\end{aligned}
$$

where $\tilde{T}$ is the trace of the matter stress-energy tensor in the Jordan frame and we linearize the equation in the second line. Hence, the effect of the nonminimal coupling is the introduction of a mass square-like term $-4 \pi \beta \tilde{T}$. Let us consider the spacetime of a single star consisting of a perfect fluid which is not heavily relativistic. In such a case, $\tilde{T}=-\tilde{\rho}+3 \tilde{p} \approx-\rho<0$. Thus, there are always negative values of $\beta^{1}$ that lead to $-4 \pi \beta \tilde{T}+m_{\phi}^{2}<0$, and the coefficient of $\phi$ acts as the square of an imaginary mass term, which causes exponential growth in time rather than oscillations. A field with imaginary mass and the consequent unstable behavior is called a tachyon. This exponential growth is the underlying reason for the instability of the $\phi=0$ solution; i.e., even though $\phi=0$ is a solution in the presence of matter, it is not stable.

An instability is intriguing, but an indefinitely growing field cannot provide us with a well-behaved theory. We desire this instability to saturate at some point and lead to a stable solution with finite $\phi \neq 0$, i.e., a star surrounded by a finite scalar field cloud. All fully nonlinear calculations so far show that the instability is indeed regularized, leading to stable scalar clouds $[13,14]$. The main reason for the regularization of the tachyon is the nonlinear terms in the full EOM that we ignored in the linearized version, which make the potential term positive for large values of $\phi$ [13]. The linearized EOM is tachyonic only in parts of the spacetime in which the stellar matter exists, $\tilde{T} \neq 0$, and it is a usual massive scalar outside per Eq. (2). Hence, the tachyonic instability is confined to a finite volume of the space, which possibly plays a role in regularization as well. The size of the region in which the tachyon exists also dictates what values of $\beta$ allow spontaneous growth, which is the reason this phenomenon is only possible for compact stars [13].

Solutions of spontaneously scalarized stars and their fully nonlinear dynamics have been studied by many groups [17-20]. One attractive feature of spontaneous scalarization is that scalar field amplitudes can achieve very high values so that the deviations from GR are not perturbative but of order unity. Thus, these theories can be easily detected with observations on strongly gravitating

\footnotetext{
${ }^{1}$ Positive $\beta$ can also lead to spontaneous scalarization of which the behavior can be different from $\beta<0$ [16]. We will concentrate on the $\beta<0$ case here.
} 
systems, such as binary mergers. More detail about the allowed values of the $\beta-m_{\phi}$ parameters and connections to observations can be found in Ref. [13].

Lastly, we note that these results are not specific to a Gaussian and can be extended to any function with an "inverse parabola" expansion $A(\phi)=1-|\beta| \phi^{2}+\cdots$.

\section{GHOST-BASED SPONTANEOUS SCALARIZATION}

Spontaneous scalarization can be seen as the introduction of a finite volume instability to GR, which is eventually regulated by nonlinear effects. We have presented the specific case of a tachyon, but the arguments for the eventual regularization of the scalar field can be also valid for other instabilities. In this section, we will show that scalarization occurs for the instability of a ghost field as well. While a tachyon occurs when there is an effective potential term with no lower bound, a ghost occurs when there is an effective kinetic term with no lower bound. A typical example is a classical Hamiltonian with a quadratic momentum term that has a negative coefficient. Energy can decrease indefinitely by increasing the magnitude of the momentum, leading to an instability.

The tachyon of the last section was introduced by a conformal scaling function $A$ which resembles the potential term in the scalar action; i.e., it was quadratic in $\phi$. This eventually leads to a mass term in the EOM which possesses the "wrong" sign and competes with $m_{\phi}$. Similarly, a ghost can be introduced by a scaling function that resembles the kinetic term

$$
\begin{gathered}
\frac{1}{16 \pi} \int d V R-\frac{1}{16 \pi} \int d V\left[2 g^{\mu \nu} \partial_{\mu} \phi \partial_{\nu} \phi+2 m_{\phi}^{2} \phi^{2}\right] \\
+S_{m}\left[\psi_{m}, A_{\partial}^{2}(\eta) g_{\mu \nu}\right], \quad \eta \equiv g^{\mu \nu} \partial_{\mu} \phi \partial_{\nu} \phi,
\end{gathered}
$$

where $A_{\partial}$ is a conformal scaling function in analogy to Eq. (1) but depends on the derivatives of the scalar field rather than the field itself. We will continue to use the notation of the Jordan and Einstein frames. Variations of the action lead to the EOM

$$
\begin{aligned}
& R_{\mu \nu}=8 \pi\left(T_{\mu \nu}-\frac{1}{2} g_{\mu \nu} T\right)+2 \partial_{\mu} \phi \partial_{\nu} \phi+m_{\phi}^{2} \phi^{2} g_{\mu \nu} \\
& \square_{g} \phi=8 \pi A_{\partial}^{4} \alpha_{\partial} \tilde{T} \square_{g} \phi+m_{\phi}^{2} \phi,
\end{aligned}
$$

where $\alpha_{\partial}=d\left(\ln A_{\partial}\right) / d \eta$. Reorganizing the scalar EOM and using $A_{\partial}(\eta)=e^{\beta_{\partial} \eta / 2}$ in analogy to $A$, we reach

$$
\left(-4 \pi A_{\partial}^{4} \beta_{\partial} \tilde{T}+1\right) \square_{g} \phi=m_{\phi}^{2} \phi .
$$

As long as $\tilde{T}$ is nonzero, there are always values of $\beta_{\partial}$ that make the coefficient of $\square_{g} \phi$ negative. This is an exact analog of spontaneous scalarization that we studied in the previous section. The kinetic term has the wrong sign now, which is the main feature of a ghost. $\phi$ tends to grow similarly to a tachyon and hence is capable of growing arbitrarily small deviations from $\phi=0$. This is still spontaneous scalarization, albeit arising from a different instability. We will call this newly devised mechanism ghost-based spontaneous scalarization to distinguish it from the previous case, which we will call tachyon-based spontaneous scalarization. We can rearrange Eq. (5) as

$$
\square_{g} \phi=\left(-4 \pi A_{\partial}^{4} \beta_{\partial} \tilde{T}+1\right)^{-1} m_{\phi}^{2} \phi=m_{\mathrm{eff}}^{2} \phi,
$$

where $m_{\text {eff }}^{2}<0$ when the field behaves like a ghost. Hence, a ghost and a tachyon behave similarly at the level of the EOM, which is another way to see the instability of the ghost.

The next crucial requirement for a successful spontaneous scalarization theory is the eventual regularization of the instability. This is accomplished by nonlinear terms and perhaps helped by the existence of the unstable EOM only in a finite volume for the case of the tachyon. The same mechanisms are also present for the ghost-based version as the form and especially the sign of $m_{\text {eff }}$ are closely related to the case of the tachyon. Thus, we also expect the ghost to be eventually regularized, causing a stable nonzero scalar field configuration. To see this explicitly, we calculate scalarized NS solutions of this theory.

We can derive the Tolman-Oppenheimer-Volkoff (TOV)like equations for a static, spherically symmetric star from the metric ansatz

$$
g_{\mu \nu} d x^{\mu} d x^{\nu}=-e^{\nu(r)} d t^{2}+\frac{d r^{2}}{1-2 \mu(r) / r}+r^{2} d \Omega^{2} .
$$

We assume NS matter to be a perfect fluid with $\tilde{T}^{\mu \nu}=$ $(\tilde{\rho}+\tilde{p}) \tilde{u}^{\mu} \tilde{u}^{\nu}+\tilde{p} \tilde{g}^{\mu \nu}$ where the energy density $\tilde{\rho}$, pressure $\tilde{p}$, and components of the fluid 4-velocity $\tilde{u}^{\alpha}$ only depend on the radial coordinate $r$. EOM reduce to the set of ordinary differential equations

$$
\begin{aligned}
x^{\prime} & =\left(8 \pi A_{\partial}^{4} \tilde{\rho}+m_{\phi}^{2} \phi^{2}+\chi^{2}\right) r-x / r \\
\nu^{\prime} & =\left[8 \pi A_{\partial}^{4} \tilde{p}-m_{\phi}^{2} \phi^{2}+(2 \gamma-1) \chi^{2}+x / r^{2}\right] r /(1-x) \\
\phi^{\prime} & =\chi / \sqrt{1-x} \\
\chi^{\prime} & =-\left[\nu^{\prime} / 2+2 / r\right] \chi+\gamma^{-1} m_{\phi}^{2} \phi / \sqrt{1-x} \\
\tilde{p}^{\prime} & =-(\tilde{\rho}+\tilde{p})\left[\nu^{\prime} / 2+2 \alpha_{\partial} \chi \chi^{\prime}\right],
\end{aligned}
$$

where $x=2 \mu / r, \chi=\psi \sqrt{1-x}, \gamma=1-8 \pi A_{\partial}^{4} \alpha_{\partial} \tilde{T}$, and ' denotes a derivative with respect to $r$. This system of equations is completed by an equation of state (EOS) $\tilde{\rho}=\tilde{\rho}(\tilde{p})$.

The sign of $\gamma$ determines the ghost nature of $\phi$ in Eq. (5). $\gamma<0$ has to be satisfied in some region of the NS if a ghost is to exist at all. $\gamma=1$ outside the NS where $\tilde{T}=0$, so any physically sensible solution that has continuous TOV variables dictates $\gamma=0$ at some radius $r_{\star}$ inside the star. 


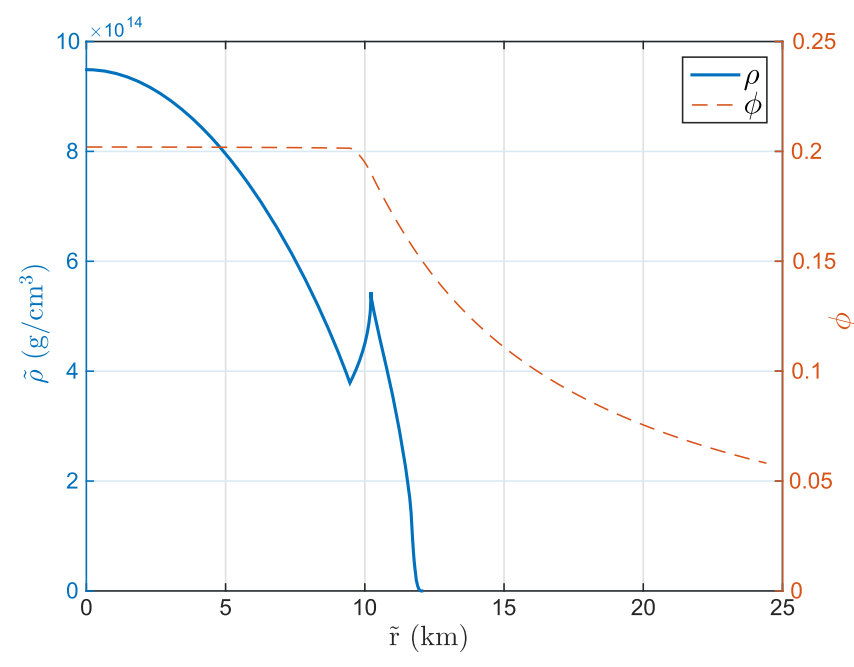

FIG. 1. The structure of a $1.81 M_{\odot}$ Arnowitt-Deser-Misner (ADM) mass NS with HB EOS [22] that is scalarized through a ghost instability for $m_{\phi}=1.6 \times 10^{-12} \mathrm{eV}$ and $\beta_{\partial}=6.7 \times$ $10^{-7} \mathrm{~km}^{-2} . \tilde{r}=A r$ is the radius in Jordan frame. The cusp is clearly visible at $\tilde{r} \approx 10 \mathrm{~km}$. The sudden change in $\tilde{\rho}$ before the cusp at $\tilde{r} \approx 9 \mathrm{~km}$ is not a cusp; derivatives of the TOV variables are large but finite at this point.

This is potentially troublesome since $\chi^{\prime}$ and $\tilde{p}^{\prime}$ diverge at this point due to the $m_{\text {eff }}^{2}=\gamma^{-1} m_{\phi}^{2}$ term. However, these divergences in the derivatives do not imply that $\chi$ or $\tilde{p}$ diverges; rather, they have cusps. More specifically, they behave as $C_{1}+C_{2}\left|r-r_{\star}\right|^{n}$ near $r_{\star}$ with $0<n<1$ and $C_{1}, C_{2}$ constants. This is also explicitly verified by our numerical solution.

A divergent derivative is problematic for standard explicit time stepping methods even when the solutions are finite and continuous. Hence, we used implicit methods implemented in the CVODE package of the SUNDIALS suite to solve Eq. (8) in order to achieve numerical stability [21]. A sample solution can be seen in Fig. 1, in which a finite scalar field cloud is present around the NS. This indicates the eventual regularization of the ghost instability in a fashion similar to the tachyon. However, as we mentioned before, a thorough understanding of the stability of this solution is only possible with time evolution, which is left to future studies. We leave time evolution and a more detailed study of the effects of different NS EOS and $m_{\phi}-$ $\beta_{\partial}$ parameters to future studies. The effect of the cusp on stationary NSs and during time evolution are especially interesting to investigate, in addition to order-of-unity deviations from GR that are the hallmark of spontaneous scalarization theories in general.

\section{GHOST-BASED SPONTANEOUS TENSORIZATION}

Just as the type of instability is not critical for spontaneous growth, recent studies have shown that the type of the field that carries the instability is not critical either; e.g., one can replace the scalar with a vector field and still observe spontaneous growth. This has been recently studied for a tachyonlike instability living on a vector field [10],

$$
\begin{aligned}
& \frac{1}{16 \pi} \int d^{4} x \sqrt{-g} R-\frac{1}{16 \pi} \int d^{4} x \sqrt{-g}\left[F^{\mu \nu} F_{\mu \nu}+2 m_{X}^{2} X^{\mu} X_{\mu}\right] \\
& \quad+S_{m}\left[\psi_{m}, A_{X}^{2}(\eta) g_{\mu \nu}\right], \quad \eta=g^{\mu \nu} X_{\mu} X_{\nu},
\end{aligned}
$$

where $F_{\mu \nu}=\nabla_{\mu} X_{\nu}-\nabla_{\nu} X_{\mu}$, and the matter terms are similar to Eq. (1). Analogy to spontaneous scalarization is manifest in the EOM

$$
\nabla_{\rho} F^{\rho \mu}=\left(-8 \pi A_{X}^{4} \alpha_{X} \tilde{T}+m_{X}^{2}\right) X^{\mu},
$$

where $\alpha_{X}(\eta)=d\left(\ln A_{X}(\eta)\right) / d(\eta)$ and $\tilde{T}_{\mu \nu}$ is the stressenergy tensor in the Jordan frame. It can be shown that the vector field can spontaneously grow for appropriate choices of $A_{X}$ leading to nontrivial vector field clouds around neutron stars. This generalization of spontaneous growth to vectors is called spontaneous vectorization, and the general term for spontaneous growth of any type of fields is named spontaneous tensorization. Close similarity of spontaneous vectorization in Eq. (9) to spontaneous scalarization strongly suggests the stability of vectorized neutron stars, but no time evolution has been performed to explicitly demonstrate this [10].

The next natural step is combining the two ideas, that is, generalizing both the type of instability and the field that carries the instability. The simplest such generalization is ghost-based spontaneous vectorization. The form of the action is straightforward when we replace the scalar with a vector and remember that $A_{\partial}$ resembled the form of the kinetic term in ghost-based spontaneous scalarization,

$$
\begin{gathered}
\frac{1}{16 \pi} \int d V R-\frac{1}{16 \pi} \int d V\left[F^{\mu \nu} F_{\mu \nu}+2 m_{X}^{2} X^{\mu} X_{\mu}\right] \\
+S_{m}\left[\psi_{m}, A_{F}^{2}\left(\eta_{F}\right) g_{\mu \nu}\right], \quad \eta_{F}=F^{\mu \nu} F_{\mu \nu} .
\end{gathered}
$$

The Jordan frame is associated with the metric $\tilde{g}_{\mu \nu}=A_{F}^{2} g_{\mu \nu}$. The EOM for the vector is

$$
\left(-8 \pi A_{F}^{4} \alpha_{F} \tilde{T}+1\right) \nabla_{\rho} F^{\rho \mu}=m_{X}^{2} X^{\mu},
$$

where $\alpha_{F} \equiv d\left(\ln A_{F}\left(\eta_{F}\right)\right) / d \eta_{F}$. This is a vector ghost for appropriate functions $\alpha_{F}$, in exact analogy to ghost-based spontaneous scalarization. All our consequent arguments about the eventual regularization and astrophysical relevance of the scalar ghost can also be repeated for the vector without significant obstacles. We will not attempt a detailed study of these issues here, but we once more see that spontaneous growth and instability regularization are relevant well beyond tachyonic instabilities of scalar fields. Establishing the stability of any solution in this theory again awaits time evolution studies. 
After scalars and vectors, the next target for spontaneous growth in the tensor hierarchy is a rank- 2 tensor representing a spin-2 particle. Let us summarize how we have been constructing theories of spontaneous growth. First, we start with the Einstein-Hilbert action and a minimally coupled field (scalar or vector), $S_{\mathrm{EH}}+S_{\text {field }}$; hence, everything is under the umbrella of GR. Second, we finalize the construction by adding matter fields that are nonminimally coupled to the Einstein-frame metric by simply scaling the metric in the matter coupling by a function of the field or its derivative,

$$
S=S_{\mathrm{EH}}+S_{\text {field }}+S_{m}\left[\psi_{m}, A^{2} \text { (field) } g_{\mu \nu}\right] .
$$

A theory of spontaneously growing rank-2 tensors is less straightforward to build, and the conventional reasoning for this hardship is as follows. The Einstein frame metric $g_{\mu \nu}$ itself is a spin-2 field, and theories of interacting spin-2 particles including massive spin-2 fields have historically been considered to be ill behaved due to their inclusion of ghosts [5]. Note that the ghosts that appear in these theories, let us call them unwanted ghosts, are not similar to the ghosts that we intentionally introduced to instigate spontaneous growth in the previous sections. Unwanted ghosts appear as a side effect of the interactions, behave as ghosts in all parts of the spacetime, and most importantly do not eventually regularize. Hence, even the first part of our construction, $S_{\mathrm{EH}}+S_{\text {field }}$, fails when the field is spin 2 (a second metric).

Even though the history of interacting spin-2 fields does not look promising for spontaneous growth at first, ghostfree theories have been successfully introduced recently, including minimal matter coupling [5-7]. Schematically, their action is in the form

$$
S=S_{\mathrm{EH}}(g)+S_{\mathrm{EH}}(f)+S_{\mathrm{int}}(f, g)+S_{m}\left[\psi_{m}, g_{\mu \nu}\right],
$$

where we have two separate Einstein-Hilbert actions for two metrics $f$ and $g$, an interaction piece which has a specific form to eliminate any ghosts while making the fields massive, and finally a minimal matter coupling to one of the metrics. This renews our hope to have spontaneous growth for spin-2 fields. By following the logic for the lower spin fields, in Eq. (14), we simply change the matter coupling into a nonminimal one with a conformal scaling,

$$
S_{m}\left[\psi_{m}, g_{\mu \nu}\right] \rightarrow S_{m}\left[\psi_{m}, A_{\text {spin-2 }}^{2}(f, g) g_{\mu \nu}\right] .
$$

An appropriate choice of $A_{\text {spin-2 }}$, as in the lower spin cases, can induce an instability in the presence of NSs. If this instability is eventually regularized, we can have a wellbehaved alternative gravity theory with spontaneously growing spin-2 fields.

The above ideas give us a road map to investigate spontaneous scalarizarion of spin-2 fields, but there is a major obstacle on this path: nonminimal matter couplings such as Eq. (15) in bigravity theories are known to reintroduce the unwanted ghosts in many cases [8]. This means, in addition to the instability we intentionally introduce for spontaneous growth, the unwanted ghost might also reappear. Hence, the first issue to check is whether the unwanted ghost is reintroduced. If it is not, then we have a novel well-behaved alternative theory of gravitation. Such a theory is interesting for connecting the idea of massive gravity and bigravity with spontaneous growth. If the ghost is reintroduced and the theory is ill behaved, this road map has to be abandoned.

We want to mention a third, more speculative, possibility. Even if the unwanted ghost reappears at the linear level, could it also be regularized by nonlinear effects similarly to the instability directly arising from the nonminimal matter coupling? Irrespective of the details, our proposed spontaneously growing rank-2 field theory behaves identically to the well-behaved, ghost-free theory in vacuum where the matter coupling is irrelevant. Thus, when considering the spacetime of an isolated NS, the unwanted ghost is confined within the volume of the star, just like the instability that is intentionally introduced to cause spontaneous growth (whether it is a tachyon or a ghost). If nonlinear terms behave as they behave in spontaneous scalarization, this finite volume unwanted ghost might also be regularized. This is a rather interesting possibility, and the nonlinear behavior of the unwanted ghost can be analyzed using techniques in massive gravity literature [5-7]. Nevertheless, we should emphasize that all our discussions on spontaneous growth of spin-2 fields are speculation before any concrete studies to test them.

\section{DISCUSSION}

The core idea of this study is the fact that instabilities in gravity theories can be regularized by nonlinear effects, especially when higher-order interactions are present and the instabilities are confined to a finite volume in space. This can render them harmless and sometimes even useful. This has been known in the case of spontaneous scalarization theories in which a tachyonic instability is regularized to form stable scalar clouds around NSs. The large deviations from GR that are caused by these clouds are an attractive target for gravitational wave observations and other tests of strong gravity. Since there is nothing specific about tachyons in terms of their growth and regularization, it is natural to expect a similar result for other instabilities. We verify this idea by showing that ghost instabilities also lead to nontrivial scalarized NS solutions. Furthermore, the type of the field that possesses the instability, scalar vs vector, is not crucial, either. The regularization mechanism of spontaneous scalarization seems to be powerful over many forms of instabilities, showing that tachyon-based spontaneous scalarization is only one member of a vast family of spontaneous growth theories. However, the stability of the solutions for these newly identified theories remains to be studied. 
Following our line of thought brought us to a theory of spontaneous growth of spin-2 fields, which also makes a strong connection to massive gravity and bigravity. Interacting spin-2 fields are plagued by unregularized ghosts that render them unphysical. Even though these ghosts can be removed by recent breakthroughs in the field, nonminimal matter couplings, such as the ones we introduce for spontaneous growth, are known to bring them back in many situations. We discussed the three main possibilities for a proposed theory of spontaneously growing spin-2 fields. First, the nonminimal matter coupling we introduce for spontaneous growth somehow does not reintroduce the unwanted ghost, and our idea is successful. Second, the ghost is reintroduced and is ill behaved in the most general case (in the nonlinear regime), meaning our proposal fails. Third, even if the unwanted ghost is reintroduced at the linear level, it is regularized at the nonlinear level by mechanisms similar to those we discussed for spontaneous growth, still leading to a well-behaved theory. The last possibility might present an opportunity to test the power of the regularization mechanisms on an instability that is not purposefully introduced. We again emphasize that our discussion of spin-2 fields, especially the third possibility, is not beyond the level of speculation before we concretely study them in future work.
Even though we concentrated on instabilities arising from conformal scalings of the matter coupling, our ideas can be applied to other couplings as well. For the specific case of scalar fields in gravity, possible terms in the Lagrangian have been classified by Horndeski and recent extensions of his work $[11,12]$. In addition to the conformal scalings in the nonminimal matter coupling, more general options like disformal and extended disformal couplings might also lead to spontaneous growth and provide novel examples of instability regularization [23]. Overall, the understanding of instabilities provides valuable insight for both formal aspects of gravity theories and observable signatures of alternative theories.

\section{ACKNOWLEDGMENTS}

Numerical calculations were performed on the Perseus cluster at Princeton University and Lüfer cluster at Koç University. Many ideas we present here are the result of our stimulating discussions with Bayram Tekin, for whom we are deeply thankful. We are also grateful for Metin Muradoğlu for his help with numerical methods for stiff ordinary differential equations. The author would like to acknowledge the contribution of the COST Action CA16104.
[1] B. P. Abbott et al. (LIGO Scientific and Virgo Collaborations), Phys. Rev. Lett. 116, 061102 (2016).

[2] B. P. Abbott et al. (LIGO Scientific and Virgo Collaborations), Phys. Rev. Lett. 116, 221101 (2016).

[3] E. Berti et al., Classical Quantum Gravity 32, 243001 (2015).

[4] C. M. Will, Living Rev. Relativity 9, 3 (2006).

[5] C. de Rham, Living Rev. Relativity 17, 7 (2014).

[6] C. de Rham, G. Gabadadze, and A. J. Tolley, Phys. Rev. Lett. 106, 231101 (2011).

[7] S. F. Hassan and R. A. Rosen, J. High Energy Phys. (2012) 126.

[8] C. de Rham, L. Heisenberg, and R. H. Ribeiro, Phys. Rev. D 90, 124042 (2014).

[9] C. Brans and R. H. Dicke, Phys. Rev. 124, 925 (1961).

[10] F. M. Ramazanoğlu, Phys. Rev. D 96, 064009 (2017).

[11] G. W. Horndeski, Int. J. Theor. Phys. 10, 363 (1974).

[12] J. Gleyzes, D. Langlois, F. Piazza, and F. Vernizzi, Phys. Rev. Lett. 114, 211101 (2015).

[13] F. M. Ramazanoğlu and F. Pretorius, Phys. Rev. D 93, 064005 (2016).
[14] T. Damour and G. Esposito-Farèse, Phys. Rev. Lett. 70, 2220 (1993).

[15] P. Chen, T. Suyama, and J. Yokoyama, Phys. Rev. D 92, 124016 (2015).

[16] R. F. P. Mendes and N. Ortiz, Phys. Rev. D 93, 124035 (2016).

[17] E. Barausse, C. Palenzuela, M. Ponce, and L. Lehner, Phys. Rev. D 87, 081506 (2013).

[18] M. Shibata, K. Taniguchi, H. Okawa, and A. Buonanno, Phys. Rev. D 89, 084005 (2014).

[19] D. Gerosa, U. Sperhake, and C. D. Ott, Classical Quantum Gravity 33, 135002 (2016).

[20] N. Sennett and A. Buonanno, Phys. Rev. D 93, 124004 (2016).

[21] A. C. Hindmarsh, P. N. Brown, K. E. Grant, S. L. Lee, R. Serban, D. E. Shumaker, and C. S. Woodward, ACM Trans. Math. Softw. 31, 363 (2005).

[22] J. S. Read, C. Markakis, M. Shibata, K. Uryū, J. D. E. Creighton, and J. L. Friedman, Phys. Rev. D 79, 124033 (2009).

[23] M. Zumalacrregui and J. Garca-Bellido, Phys. Rev. D 89, 064046 (2014). 